\title{
Investigation of hemodynamics in an in vitro system simulating left ventricular support through the right subclavian artery using 4-dimensional flow magnetic resonance imaging
}

\author{
Bernd Jung, PhD, ${ }^{\mathrm{a}}$ Christoph Müller, BSc, ${ }^{\mathrm{b}}$ Waltraud Buchenberg, Dipl Phys, ${ }^{\mathrm{b}}$ Michael Ith, PhD, ${ }^{\mathrm{a}}$ \\ David Reineke, MD, ${ }^{\mathrm{c}}$ Friedhelm Beyersdorf, $\mathrm{MD},{ }^{\mathrm{d}}$ and Christoph Benk, $\mathrm{PhD}^{\mathrm{d}}$
}

\begin{abstract}
Objectives: Left ventricular assist devices are an important treatment option for patients with heart failure alter the hemodynamics in the heart and great vessels. Because in vivo magnetic resonance studies of patients with ventricular assist devices are not possible, in vitro models represent an important tool to investigate flow alterations caused by these systems. By using an in vitro magnetic resonance-compatible model that mimics physiologic conditions as close as possible, this work investigated the flow characteristics using 4-dimensional flow-sensitive magnetic resonance imaging of a left ventricular assist device with outflow via the right subclavian artery as commonly used in cardiothoracic surgery in the recent past.
\end{abstract}

Methods: An in vitro model was developed consisting of an aorta with its supraaortic branches connected to a left ventricular assist device simulating the pulsatile flow of the native failing heart. A second left ventricular assist device supplied the aorta with continuous flow via the right subclavian artery. Four-dimensional flow-sensitive magnetic resonance imaging was performed for different flow rates of the left ventricular assist device simulating the native heart and the left ventricular assist device providing the continuous flow. Flow characteristics were qualitatively and quantitatively evaluated in the entire vessel system.

Results: Flow characteristics inside the aorta and its upper branching vessels revealed that the right subclavian artery and the right carotid artery were solely supported by the continuous-flow left ventricular assist device for all flow rates. The flow rates in the brain-supplying arteries are only marginally affected by different operating conditions. The qualitative analysis revealed only minor effects on the flow characteristics, such as weakly pronounced vortex flow caused by the retrograde flow via the brachiocephalic artery.

Conclusions: The results indicate that, despite the massive alterations in natural hemodynamics due to the retrograde flow via the right subclavian and brachiocephalic arteries, there are no drastic consequences on the flow in the brain-feeding arteries and the flow characteristics in the ascending and descending aortas. It may be beneficial to adjust the operating condition of the left ventricular assist device to the residual function of the failing heart. (J Thorac Cardiovasc Surg 2015;150:200-7)

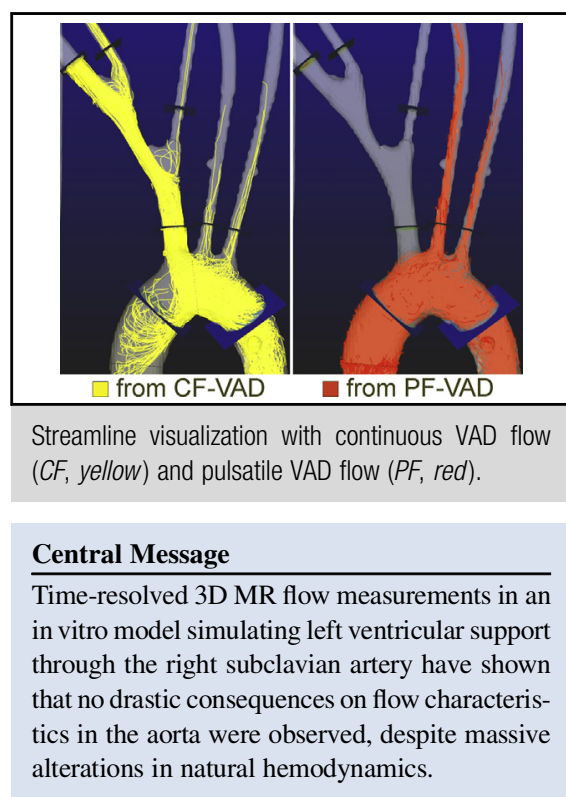

\section{Perspective}

By using an in vitro MR-compatible model system, the flow characteristics caused by a VAD blood supply via the right subclavian artery were investigated under different VAD operating conditions as measured by 4D phasecontrast MRI. Despite the alterations in natural hemodynamics by retrograde flow, no drastic consequences on aortic hemodynamics were observed, as supported by recent experiences with partial-support devices for long-term support in patients with chronic heart failure.

See Editorial Commentary page 207.
From the Institute of Diagnostic, Interventional and Pediatric Radiology, ${ }^{\mathrm{a}}$ University Hospital Bern, Bern, Switzerland; Department of Radiology, ${ }^{\mathrm{b}}$ Medical Physics, University Hospital, Freiburg, Germany; Department of Cardiovascular Surgery, ${ }^{c}$ University Hospital Bern, Bern, Switzerland; and Cardiovascular Surgery, ${ }^{\mathrm{d}}$ Heart Center Freiburg University, Freiburg, Germany.

Funded by Deutsche Forschungsgemeinschaft Grant JU 2687/9-1.

Received for publication Nov 18, 2014; revisions received Jan 26, 2015; accepted for publication Feb 19, 2015; available ahead of print April 1, 2015.
Address for reprints: Bernd Jung, $\mathrm{PhD}$, Institute of Diagnostic, Interventional and Pediatric Radiology, University Hospital Bern, 3010 Bern, Switzerland (E-mail: bernd.jung@insel.ch).

$0022-5223 / \$ 36.00$

Copyright (ㄷ 2015 by The American Association for Thoracic Surgery

http://dx.doi.org/10.1016/j.jtcvs.2015.02.048 


\section{Abbreviations and Acronyms}

$\mathrm{CFD}=$ Computational Fluid Dynamics

$\mathrm{CF}-\mathrm{VAD}=$ constant flow ventricular assist device

$4 \mathrm{D} \quad=4$-dimensional

LVAD $=$ left ventricular assist device

MR = magnetic resonance

MRI = magnetic resonance imaging

$\mathrm{PF}-\mathrm{VAD}=$ pulsatile flow ventricular assist device

$3 \mathrm{D}=3$-dimensional

$\mathrm{VAD}=$ ventricular assist device

Video clip is available online.

Left ventricular assist devices (LVADs) have become an important tool for therapy in patients with heart failure to bridge the time until heart transplantation or as a permanent solution. ${ }^{1-5}$ The lack of donors and promising results of LVAD therapy have led to an increased use of these fully implantable pumps (axial or centrifugal) in patients for long-term support or destination therapy. However, some potentially lethal and devastating complications remain after LVAD implantation, including thrombus formation and embolization, valve dysfunction (eg, aortic insufficiency or valve thrombosis), infections, and device failures. ${ }^{6-11}$ To prevent thromboembolization, most devices require a sophisticated anticoagulation regimen, which may result in further complications.

Various types of ventricular assist devices (VADs) are available with a variety of connection possibilities to the cardiovascular system providing cardiac support with continuous or pulsatile flow. Optimal positioning of the inflow and outflow cannulas is essential for an effective unloading of the failing heart and to guarantee an adequate blood supply to the organs. ${ }^{12,13}$ However, VADs can result in altered hemodynamics that may cause a higher thrombotic risk or the development of heart valve defects..$^{6-11}$ Therefore, the design of the cannula and the proper connection to the cardiovascular system, as well as operating conditions of the VAD, are crucial with respect to the hemodynamics in the heart and the great vessels to avoid valve dysfunction or thromboembolization, for example, due to a stasis in the inflow area of an apex cannula.

Although VADs are successfully applied in patients with heart failure, only limited knowledge is available with respect to the impact of VADs on the hemodynamics. A few studies have been presented using 2-dimensional and 3-dimensional (3D) Computational Fluid Dynamics (CFD) models to investigate the effects of VADs on ventricular and aortic branch flow patterns. In particular, a few studies reported on the use of an aortic branch and left ventricle model to evaluate the effect of different inflow and outflow cannula tip geometries by using CFD or particle image velocimetry. ${ }^{14-18}$ Nevertheless, applicable and valid results are hindered by complex numeric models with extremely high computational demands or by the limited dimensionality and the limited optical access of particle image velocimetry measurements. Oversimplified CFD models or choosing simplified boundary conditions can lead to a loss of precision in the simulations and thus to unreliable results.

Recently, the assessment of flow characteristics inside a paracorporeal pneumatically driven VAD by magnetic resonance imaging (MRI) techniques has been proposed to provide insights into the mechanisms underlying the high rate of thromboembolic events after implantation of a VAD. ${ }^{19}$ It has been shown that flow-sensitive time-resolved 3D MRI (4-dimensional [4D]-flow MRI) permits the detailed analysis of local and global flow dynamics in a realistic environment. For instance, VAD operating conditions and the valve design within the VAD strongly affect the flow pattern, such as vortex flow inside the VAD at typical locations of thrombus formation. ${ }^{20}$ These promising results show the potential to enhance the understanding of the mechanisms underlying possible thrombus formation inside a VAD and the effect of different system adjustments. It also has been demonstrated that rapid prototyping of in vitro models of a thoracic aorta can be used to simulate geometric changes, such as the introduction of an aortic stenosis to investigate the impact of the stenosis grade on the hemodynamics in the entire aorta ${ }^{21}$ or to simulate different cannula positions in the ascending or descending aorta of an LVAD. ${ }^{22}$

The purpose of the current study was to investigate the flow characteristics in a realistic in vitro model system simulating the blood supply of the thoracic aorta and the supra-aortic branches with an LVAD connected to the right subclavian artery, as commonly used as partial ventricular support in cardiothoracic surgery in the recent past. ${ }^{23}$ The output of the remaining cardiac function, as simulated by an magnetic resonance (MR)-compatible VAD providing pulsatile flow, and the flow rate of the LVAD support were systematically varied to qualitatively and quantitatively evaluate the impact of such boundary conditions on aortic and supra-aortic flow patterns.

\section{MATERIALS AND METHODS}

\section{In Vitro Model System Setup}

As schematically illustrated in Figure 1, the MR-compatible in vitro model system consisted of a flow circuit with a pneumatically driven pulsatile flow VAD (PF-VAD) with a $60 \mathrm{~mL}$ pump volume (MEDOS, Stolberg, Germany) mimicking the native (failing) heart, which was attached to a model of the thoracic aorta made of synthetic resin (Biresin U1404; SIKA GmbH, Stuttgart, Germany) to simulate the elastic property of the human aorta. ${ }^{22}$ Its shape was modeled on the basis of a 3D data set of a healthy human. The aorta model also included the supra-aortic branches 


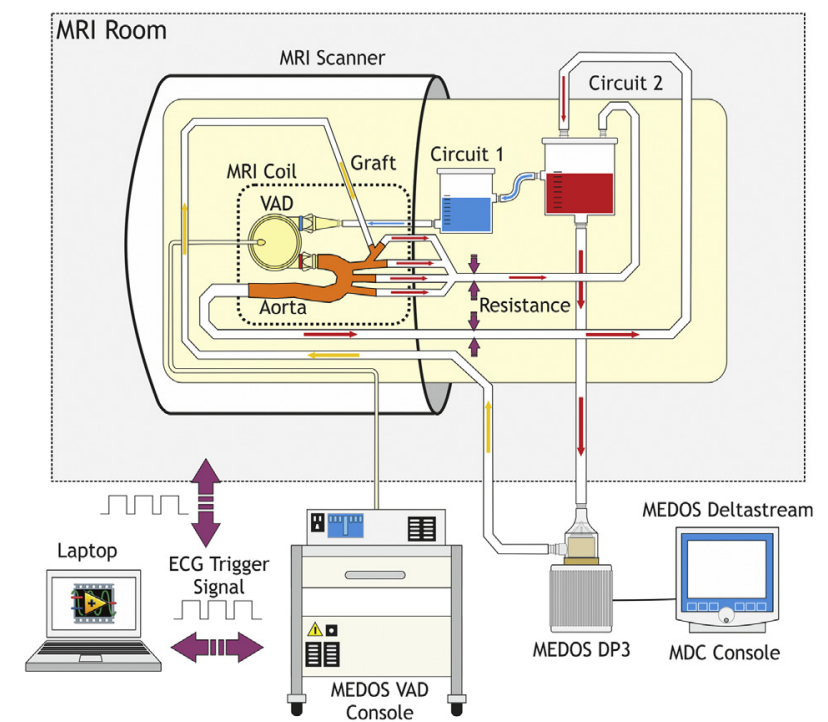

FIGURE 1. Schematic of the experimental setup. MRI, Magnetic resonance imaging; $V A D$, ventricular assist device; $E C G$, electrocardiographic.

with the brachiocephalic artery splitting in the right subclavian artery and the right carotid artery.

A diagonal pump (Deltastream DP 3; MEDOS Medizintechnik AG, Stolberg, Germany) providing a constant flow VAD (CF-VAD) was connected to the right subclavian artery and therefore supplied the aorta via a retrograde flow through the brachiocephalic artery. This CF-VAD was positioned outside the scanner room because of its non-MR-compatibility and connected with long polyvinylchloride tubing (8-m length) to the model.

Two linked circuits with 2 reservoirs were placed between the inlet and the outlet of the in vitro model. Circuit 1 as preload reservoir was connected with the PF-VAD; circuit 2 as afterload reservoir received the liquid from the supra-aortic vessels and the aorta and supplied the CF-VAD. Thus, the pressure and the resistance of the aorta could be kept stable and did not influence the preload and afterload conditions. ${ }^{21}$

\section{Experimental Conditions}

Measurements were performed with different conditions of the PF-VAD and the CF-VAD. The PF-VAD simulating the native heart was operated in 3 modes: (1) complete filling and emptying of the PF-VAD as a baseline/ reference; (2) reduced cardiac output simulating a reduction of flow volume of approximately $25 \%$ (corresponding to a cardiac output of $\sim 45 \mathrm{~mL}$ per cycle) of the baseline cardiac output ("reduced CO \#1"); and (3) reduced cardiac output of approximately $50 \%$ corresponding to a cardiac output of approximately $30 \mathrm{~mL}$ per cycle ("reduced $\mathrm{CO} \# 2$ "). All experiments were performed with a frequency of 60 beats/min.

The CF-VAD was operated with 3 different flow rates of 2, 3, and $4 \mathrm{~L} / \mathrm{min}$ for each of the 2 reduced cardiac output conditions. To simulate realistic conditions, $30 \%$ to $35 \%$ of the flow volume from the ascending aorta left the supra-aortic branches, and $65 \%$ to $70 \%$ was flowing through the descending aorta. These conditions were initially adjusted during baseline measurement using clips (see "Resistance" in Figure 1) that were not modified for the other scans. The flow rates of the CF-VAD were adjusted at the Deltastream console (MEDOS Medizintechnik AG) using an ultrasonic flow probe.

\section{Magnetic Resonance Experiments}

MR imaging experiments were performed on a 3T system (Magnetom TRIO, Siemens, Erlangen, Germany) using a 12-channel thorax coil. A prospectively gated time-resolved $3 \mathrm{D}$ phase-contrast sequence $(\mathrm{TR}=5.3$ ms) with 3-directional velocity encoding and a velocity sensitivity of 150 $\mathrm{cm} / \mathrm{s}$ in all spatial dimensions was applied to assess 3D hemodynamics for all different boundary conditions. Data were acquired with an isotropic spatial resolution of $1.4 \mathrm{~mm}$ and a temporal resolution of $42.4 \mathrm{~ms}$, resulting in 23 time frames within 1 cardiac cycle. An additional scan without flow was performed that was subtracted from the flow scans to correct for Eddy current-related phase offsets.

A mixture of $60 \%$ distilled water and $40 \%$ glycerol representing the kinematic viscosity $\left(\nu=0.003 \mathrm{~m}^{2} / \mathrm{s}\right)$ and density of blood was used as a blood substitute. ${ }^{15}$ To increase signal-to-noise ratio, the fluid was doped with a gadolinium-based contrast agent (Gadovist; Bayer Healthcare, Leverkusen, Germany) at a concentration of $4.0 \mathrm{mmol} / \mathrm{L}$. The flip angle providing the optimal signal-to-noise ratio (Ernst angle) was determined in a prescan and therefore set to $40^{\circ}$. By using a home-built software tool (LabVIEW, National Instruments Corp, Austin, Tex), an electrocardiographic trigger signal was generated synchronously to the MEDOS PF-VAD console and sent to the MRI scanner to synchronize the segmented time-resolved measurements with the MR-compatible PF-VAD.

\section{Data Analysis}

In a first step, noise masking and anti-aliasing of phase difference images were performed followed by a calculation of a 3D angiogram (phase-contrast MR angiography), which was visualized as a 3D isosurface. As illustrated in Figure 2, 9 planes were positioned at the following locations: lower (plane 1) and upper (plane 2) ascending aorta, proximal descending aorta (plane 3), brachiocephalic artery (plane 4), inlet of the CF-VAD to the right subclavian artery (plane 5), right subclavian artery (plane 6), right carotid artery (plane 7), left carotid artery (plane 8), and left subclavian artery (plane 9). All planes were manually placed using a 3D visualization software (EnSight; CEI, Apex, NC) and carefully angulated orthogonal to the respective vessels of interest.

For quantification of flow velocities, all planes were imported into a home-built analysis tool programmed in MATLAB (The Mathworks, Inc, Natick, Mass). This tool allowed for manual vessel lumen contour segmentation and velocity quantification for each time frame. For each analysis plane, the flow rate (liters/minute) and the amount of retrograde flow (\% per heart beat) were quantified. In addition, 3D visualization of the measured time-resolved and 3-directional flow velocities was used. Visualization of aortic hemodynamics included the calculation of time-resolved $3 \mathrm{D}$ particle traces and streamlines emitted from planes 1 and 5 . The resulting traces can be viewed dynamically and depict the spatial and temporal dynamics of aortic flow over the cardiac cycle. The resulting traces were color-coded according to their origin to visualize mixing of flow streams originating in the ascending aorta, that is, from the PF-VAD, and the inlet of the CF-VAD.

\section{RESULTS}

According to the scheme in Figure 2, flow time curves are shown for 5 selected planes (ascending aorta, plane 1; brachiocephalic artery, plane 4; right subclavian artery, plane 6; right carotid artery, plane 7; left subclavian artery, plane 9) for the reference scan and 2 scans with a reduced cardiac output of the PF-VAD, the first with $45 \mathrm{~mL}$ (reduced CO \#1) and a CF-VAD support rate of $3 \mathrm{~L} / \mathrm{min}$ and the second with $30 \mathrm{~mL}$ (Reduced CO \#2) and a CF-VAD support rate of $4 \mathrm{~L} / \mathrm{min}$. The retrograde flow over the entire cardiac cycle can be clearly seen in the brachiocephalic artery (plane 4). During systole, which ranges in the setup from approximately 250 to $700 \mathrm{~ms}$ because of trigger reasons, the retrograde flow coming from the CF-VAD is reduced because of the counter pressure from the PF-VAD. The 


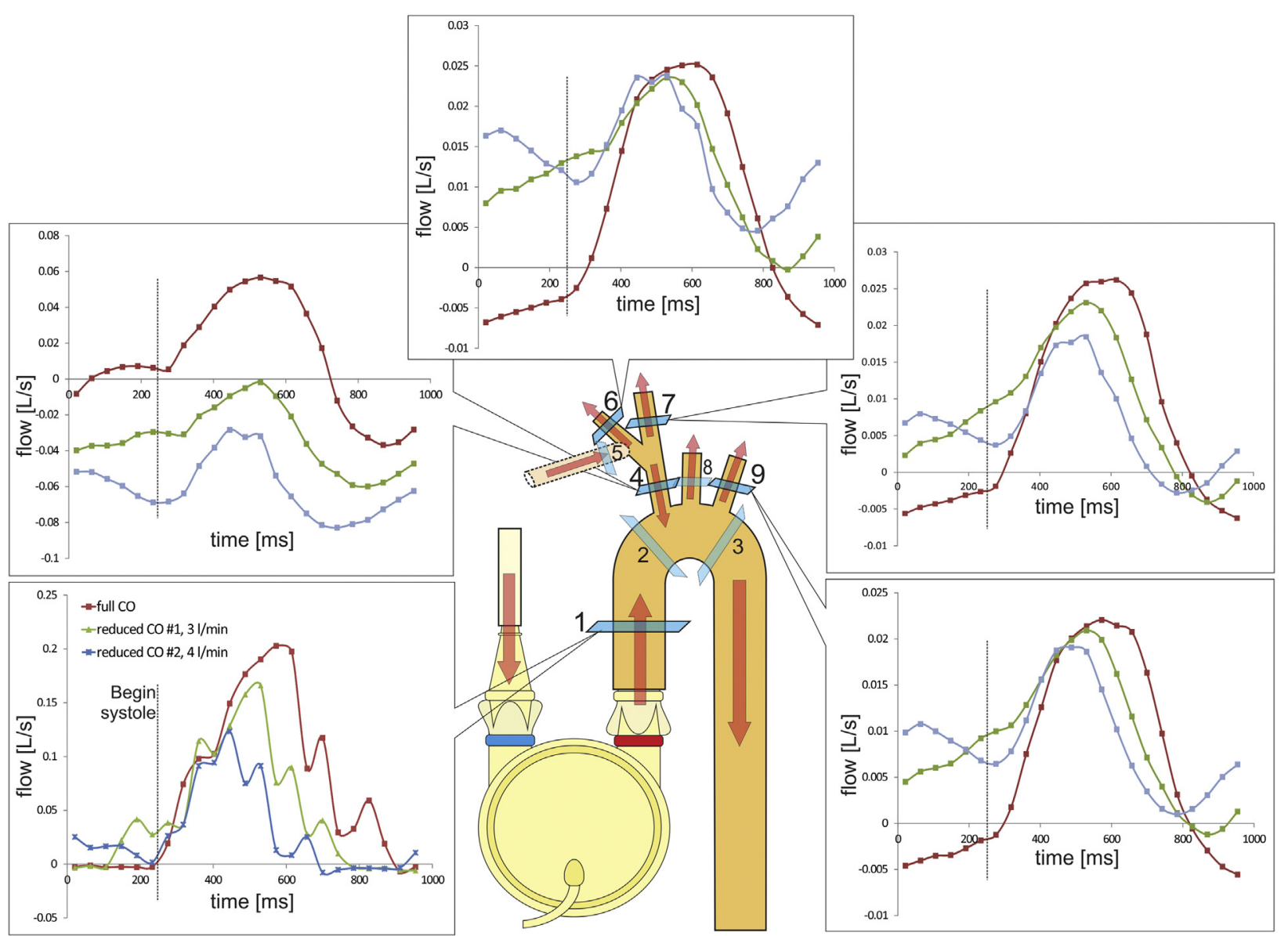

FIGURE 2. Flow-time curves in 5 selected planes for the baseline scan (full CO), the reduced CO \#1 scan with $3 \mathrm{~L} / \mathrm{min}$ support rate, and the reduced CO \#2 scan with $4 \mathrm{~L} / \mathrm{min}$ support rate. $\mathrm{CO}$, Cardiac output.

peak flow rates in the supra-aortic branches (planes 6,7, and 9) for a reduced cardiac output and an operating CF-VAD are reduced compared with the reference scan, whereas the flow rates during diastole clearly increase with increasing support rates of the CF-VAD, yielding a slightly increased net flow over the cardiac cycle in the brain-feeding arteries (Table 1).

The values for the total flow and the amount of retrograde flow for all 9 slices are summarized in Table 1, shown for the reference scan and the scans with a reduced PF-VAD output, each of them listed for the different CF-VAD operating conditions $(2,3$, and $4 \mathrm{~L} / \mathrm{min})$.

As indicated by the flow rates in the ascending aorta (plane 1 or 2) and descending aorta (plane 3), for the baseline scan it can be observed that approximately $35 \%$ of the total flow from the PF-VAD was flowing through the supraaortic branches (sum of planes 4,8 , and 9). The total flow was approximately zero with no CF-VAD support (plane 5) as expected, but systematically higher than the provided 2, 3, and $4 \mathrm{~L} / \mathrm{min}$ of the CF-VAD. Beside the inverted flow in the brachiocephalic artery (plane 4), the CF-VAD caused a decrease of the total flow in the ascending aorta (plane 1) and an increase of the total flow in the descending aorta (plane 3), both effects more pronounced the higher the flow rate supplied by the CF-VAD. Furthermore, the CFVAD caused an increase of the total flow up to $100 \%$ (mean $70 \%$, ie, averaged over all reduced cardiac output values in Table 1) for the highest support rate compared with the baseline scan in the right subclavian artery (plane 6). In contrast, a far less-pronounced increase in flow rates between the baseline and the CF-VAD-supported scans was revealed for the right (plane $7,-10 \%$ to $34 \%$; mean, $14 \%$ ) and left (plane $8,11 \%-46 \%$; mean, $26 \%$ ) carotid artery and the left subclavian artery (plane 9, 17\%-68\%; mean, 44\%). This increase is less pronounced for a more reduced remaining function of the native heart (ie, of the PF-VAD).

No or only minor retrograde flow was observed in the descending aorta (plane 3) and in the supra-aortic branches (planes 6, 7, 8, and 9) for both CF-VAD support rates and both different cardiac output conditions. The amount of retrograde flow in the ascending aorta remained small (up to a maximum of $4 \%$ ) for all operating conditions, indicating that the primary flow was directed toward the descending aorta, which is also corroborated by the streamline visualization in Figure 3. 
TABLE 1. Total and retrograde flow of baseline and pulsatile flow ventricular assist device with reduced cardiac output (reduced cardiac output \#1 and \#2) combined with 2 different support rates of the constant flow ventricular assist device ( 2 and $4 \mathrm{~L} / \mathrm{min})$

\begin{tabular}{|c|c|c|c|c|c|c|c|}
\hline & \multirow{2}{*}{$\frac{\text { Full CO }}{\text { No CF-VAD }}$} & \multicolumn{3}{|c|}{ Reduced CO \#1 } & \multicolumn{3}{|c|}{ Reduced CO \#2 } \\
\hline & & $2 \mathrm{~L} / \mathrm{min}$ & $3 \mathrm{~L} / \mathrm{min}$ & $4 \mathrm{~L} / \mathrm{min}$ & $2 \mathrm{~L} / \mathrm{min}$ & $3 \mathrm{~L} / \mathrm{min}$ & $4 \mathrm{~L} / \mathrm{min}$ \\
\hline \multicolumn{8}{|l|}{ Plane 1} \\
\hline Total flow (L/min) & 3.90 & 2.98 & 2.67 & 2.42 & 2.31 & 1.93 & 1.66 \\
\hline Retrograde flow $(\%)$ & 1.5 & 1.7 & 2.7 & 3.0 & 1.3 & 3.2 & 4.0 \\
\hline \multicolumn{8}{|l|}{ Plane 2} \\
\hline Total flow (L/min) & 3.92 & 2.87 & 2.66 & 2.36 & 2.20 & 1.95 & 1.74 \\
\hline Retrograde flow (\%) & 1.9 & 1.6 & 1.0 & 0.5 & 1.8 & 1.2 & 1.4 \\
\hline \multicolumn{8}{|l|}{ Plane 3} \\
\hline Total flow (L/min) & 2.61 & 3.10 & 3.73 & 4.20 & 2.67 & 3.09 & 3.91 \\
\hline Retrograde flow (\%) & 3.0 & 0.0 & 0.0 & 0.0 & 0.0 & 0.0 & 0.0 \\
\hline \multicolumn{8}{|l|}{ Plane 4} \\
\hline Total flow (L/min) & 0.66 & -1.29 & -1.95 & -3.23 & -1.25 & -2.26 & -3.52 \\
\hline Retrograde flow $(\%)$ & 29.0 & 92.2 & 100.0 & 100.0 & 95.6 & 100.0 & 100.0 \\
\hline \multicolumn{8}{|l|}{ Plane 5} \\
\hline Total flow (L/min) & -0.05 & 2.43 & 3.20 & 4.75 & 2.28 & 3.69 & 5.00 \\
\hline Retrograde flow (\%) & 52.6 & 0.0 & 0.0 & 0.0 & 0.0 & 0.0 & 0.0 \\
\hline \multicolumn{8}{|l|}{ Plane 6} \\
\hline Total flow (L/min) & 0.39 & 0.60 & 0.69 & 0.76 & 0.43 & 0.69 & 0.81 \\
\hline Retrograde flow (\%) & 19.9 & 3.9 & 0.1 & 0.0 & 3.2 & 0.0 & 0.0 \\
\hline \multicolumn{8}{|l|}{ Plane 7} \\
\hline Total flow (L/min) & 0.41 & 0.51 & 0.50 & 0.55 & 0.42 & 0.46 & 0.37 \\
\hline Retrograde flow (\%) & 17.0 & 6.5 & 5.5 & 3.3 & 5.8 & 3.2 & 5.1 \\
\hline \multicolumn{8}{|l|}{ Plane 8} \\
\hline Total flow (L/min) & 0.37 & 0.45 & 0.47 & 0.54 & 0.41 & 0.47 & 0.46 \\
\hline Retrograde flow (\%) & 14.6 & 4.2 & 1.3 & 0.0 & 2.5 & 0.0 & 0.0 \\
\hline \multicolumn{8}{|l|}{ Plane 9} \\
\hline Total flow (L/min) & 0.35 & 0.48 & 0.53 & 0.59 & 0.41 & 0.50 & 0.52 \\
\hline Retrograde flow $(\%)$ & 16.8 & 4.4 & 1.0 & 0.0 & 2.8 & 0.2 & 0.0 \\
\hline
\end{tabular}

Pressure values at the bottom correspond to settings on the MEDOS VAD console (Stolberg, Germany). Full CO: Psys $=280 \mathrm{~mm} \mathrm{Hg}$; Pdias $=-35 \mathrm{~mm} \mathrm{Hg}$. Reduced CO (1): Psys $=200 \mathrm{~mm} \mathrm{Hg}$; Pdias $=-30 \mathrm{~mm} \mathrm{Hg}$. Reduced CO (2): Psys $=160 \mathrm{~mm} \mathrm{Hg}$; Pdias $=-25 \mathrm{~mm} \mathrm{Hg}$. CO, Cardiac output; CF-VAD, constant flow ventricular assist device.

As expected, the flow in the brachiocephalic artery (plane 4) for the higher CF-VAD support rates ( 3 and $4 \mathrm{~L} / \mathrm{min})$ is fully directed in a retrograde manner over the cardiac cycle. A minor remaining flow $(\sim 4 \%-8 \%)$ upstream caused by the PF-VAD is still visible for the support of $2 \mathrm{~L} / \mathrm{min}$.

The sum of the flow rates PF-VAD + CF-VAD depict the total flow $\mathrm{VAD}_{\text {total }}$ from the $\mathrm{VAD}$ and the native heart. VAD $_{\text {total }}$ for a CF-VAD support rate of $2 \mathrm{~L} / \mathrm{min}+45 \mathrm{~mL}$ PF-VAD output and a CF-VAD support rate of $3 \mathrm{~L} / \mathrm{min}+$ $30 \mathrm{~mL}$ PF-VAD output result in a comparable $\mathrm{VAD}_{\text {total }}$ of approximately $4.8 \mathrm{~L} / \mathrm{min}$. The same counts for a CF-VAD support rate of $3 \mathrm{~L} / \mathrm{min}+45 \mathrm{~mL}$ PF-VAD output and a CF-VAD support rate of $4 \mathrm{~L} / \mathrm{min}+30 \mathrm{~mL}$ PF-VAD output $\left(\mathrm{VAD}_{\text {total }} \approx 5.8 \mathrm{~L} / \mathrm{min}\right.$ ). Noticeably, the flow rates in all brain-feeding arteries (planes 6, 7, 8, and 9) are slightly affected by CF-VAD conditions when comparing the flow rates of the scenarios described earlier. As expected, only plane 6 reveals a more pronounced enhancement of the flow rate as the CF-VAD support increases.

Figure 3 shows a streamline visualization of 2 different conditions, in the upper row with a moderately reduced PF-VAD output ("Reduced CO \#1") and CF-VAD support of $2 \mathrm{~L} / \mathrm{min}$ and in the lower row with a more reduced PFVAD output (Reduced CO \#2) and CF-VAD support of 4 $\mathrm{L} / \mathrm{min}$. Streamlines in the left graphs originated from the CF-VAD (plane 5, yellow), demonstrating blood flow partly down the ascending aorta against the actual flow direction, and from the PF-VAD (plane 1, red). The right subclavian artery (plane 6) and the right carotid artery (plane 7) are solely supported by the CF-VAD (upper row), whereas the left carotid artery (plane 8) and the left subclavian artery (plane 9) are mainly supported by the PF-VAD and a smaller portion by the CF-VAD with a higher support rate (lower row). The qualitative analysis revealed only minor effects on the flow characteristics, such as vortex flow or pronounced helical flow. As shown in the left graphs of Figure 3, some moderate flow vortices occur upstream and downstream of the aorta close to the location where the flow stream down the brachiocephalic artery strikes the inner curvature of the aortic arch (arrows) (Video 1). These characteristics were slightly more pronounced for a higher flow rate supplied by the CF-VAD. However, it is assumed that these effects do not play a significant role with respect to valve dysfunction or thromboembolization. 


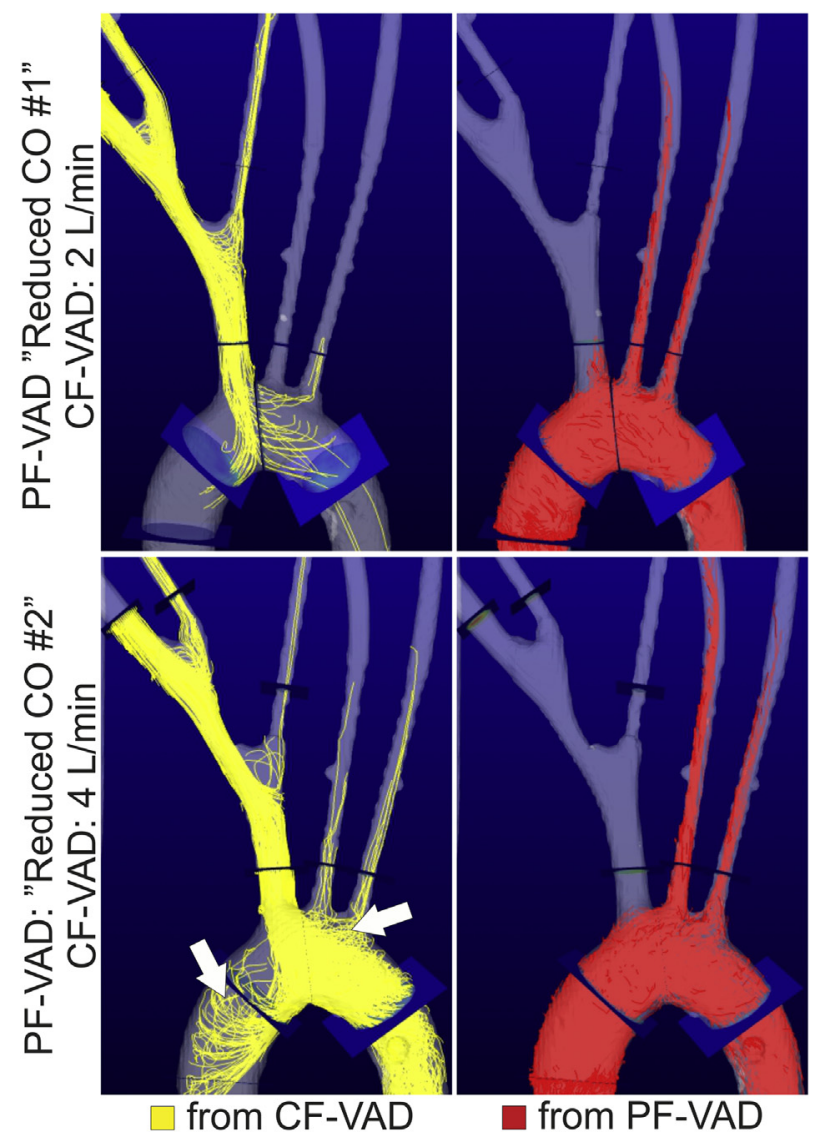

FIGURE 3. Streamline visualization color-coded according to the origin: in the left column from the CF-VAD (plane 5, yellow) and in the right column from the PF-VAD (plane 1, red). $P F-V A D$, Pulsatile flow ventricular assist device; $C O$, cardiac output; $C F-V A D$, continuous flow ventricular assist device.

\section{DISCUSSION}

Mechanical circulatory support has become a wellestablished therapy for patients with congestive heart failure. In the past years, 2 types of routine surgical approaches have been used, including a VAD that pumps blood from the left ventricular apex to the (ascending or descending) aorta or from the left atrium to the right subclavian artery. The latter is implanted using a minimally invasive technique via a small right-sided thoracotomy with an inflow cannula in the left atrium and the outflow graft connected to the right subclavian artery. ${ }^{24,25}$ However, such a procedure was expected to have a significant impact on the cardiovascular flow dynamics. In particular, outflow into the right subclavian artery resulting in retrograde supply of the aorta via the brachiocephalic artery massively changes the natural hemodynamics.

In this study, a complex in vitro model simulating an LVAD-based constant blood support via the right subclavian artery including a failing heart simulated by an MR-compatible paracorporeal LVAD was successfully established. The major aim was to qualitatively and quantitatively investigate the impact of this LVAD support on the flow pattern of the proximal aorta and the brainsupplying arteries.

The investigation of the flow characteristics inside the aorta and its upper branching vessels revealed that the right subclavian artery and the right carotid artery were solely supported by the CF-VAD for all operating conditions (2, 3 , and $4 \mathrm{~L} / \mathrm{min}$ ), whereas the descending aorta was supported by both the PF-VAD, mimicking the native failing heart, and the CF-VAD. The flow rates in all brainfeeding arteries (planes 7, 8, and 9) are only moderately affected by CF-VAD conditions. The retrograde flow in the ascending aorta slightly increases with higher flow rates provided by the CF-VAD. However, with a range of $0.5 \%$ to $4 \%$, these values are still small, and thus the impact of the retrograde flow on the functionality of the aortic valve is presumably of minor relevance. In addition, the blood circulation of the coronary arteries may be improved. On the other hand, the fractions of blood flowing from the brachiocephalic artery into the ascending aorta or toward the descending aorta may depend on the individual geometry of the aorta and brachiocephalic artery. In this case, it may be beneficial to carefully adjust the operating condition of the CF-VAD to the residual function of the failing heart to minimize the amount of blood flow from the brachiocephalic artery down the ascending aorta and therefore minimize additional burden from the CF-VAD on the failing heart and the valve function. However, this needs to be investigated in the future using information about geometries obtained from different individual subjects.

The clear decrease of the flow rates in the ascending aorta (ie, a decrease of left ventricular flow) with increasing support of the CF-VAD is in accordance with the results obtained from a study using a computational model and animal experiments. ${ }^{26}$ The increase of the flow rate in the right subclavian artery (plane 6) of up to approximately $75 \%$ (for the rather realistic conditions of $\sim 45 \mathrm{~mL}$ stroke volume for the PF-VAD (Reduced CO \#1) and $3 \mathrm{~L} / \mathrm{min}$ supply rate of the CF-VAD resulting in a cardiac output of $\sim 6$ $\mathrm{L} / \mathrm{min}$ ) leads to an "over-perfusion" of the right upper extremity, which is in accordance with observations in patients. The retrograde flow down the right subclavian artery somewhat limits/blocks the outflow of the right carotid because of the geometry (change of flow direction in the bifurcation). Therefore, the flow rate in plane 6 is higher compared with plane 7 (Figure 2, green and blue curves), whereas the flow rates are identical for the "full cardiac output" scan without support from the CF-VAD (Figure 2, red curves). Only minor effects caused by the retrograde flow via the subclavian artery could be observed in the qualitative analysis of flow patterns. Because patients with VADs cannot undergo an MRI scan, flow data of patients acquired with Doppler ultrasound could be used for a comparison of in vitro and in vivo results. 
The flow rates obtained by the MR scans clearly deviated (up to $25 \%$ higher) from the settings adjusted at the diagonal pump (CF-VAD). In addition, because of the counter-pressure of the PF-VAD, we observed beat-to beat variations of approximately $10 \%$ as measured with the ultrasonic flow probe. These deviations disappeared when the PF-VAD was switched off. Furthermore, flow rates from the PF-VAD as measured in planes 1 and 2 clearly decreased with a higher CF-VAD support rate (20\%-25\% from $2-4 \mathrm{~L} / \mathrm{min}$ ).

\section{Study Limitations}

One major limitation of this study is that it cannot be concluded how far the setup mimics in vivo physiology. We sought to simulate the arterial circulation while adjusting parameters to physiologic conditions as close as possible, such as flow rates, fluid viscosity, and geometry; however, several assumptions and simplifications have been made that may have an impact on the results, which are challenging to quantify. For instance, the in vitro model is different from the in vivo condition in some aspects, such as the inflow angle of the CF-VAD or the wall material. Although the flows in the model appear physiologic, we were not able to calculate with these data how the pressures inside the model deviate from in vivo conditions. In addition, we only adjusted the resistance for all supra-aortic branches together and not separately. However, because each vessel (in vivo) has slightly different diameters and narrows differently, the question is raised how to optimally adjust the resistance for each vessel; the subtle differences are complex to be simulated and evaluated in a model system. Moreover, 2 reservoirs were used to keep flow rates, resistance, and pressure conditions within the phantom stable and to avoid variations in the preload and afterload conditions. In our experience with only 1 reservoir, these settings are more difficult to adjust, and outgassing the circulating flow volume is more efficient with 2 reservoirs. The investigation of how geometry, resistance, pressures, reservoirs, and wall material influence hemodynamics in the model was beyond the scope of this article and may open up future investigations in terms of the optimization of such complex model systems.

In addition to the fact that the overall performance of the PF-VAD does not behave exactly like a human heart, the duration of systole with approximately 450 to $500 \mathrm{~ms}$ is clearly longer compared with an in vivo duration $(\sim 300$ $\mathrm{ms}$ ) for an RR interval of approximately 1 second; consequently, this results in a shorter diastole in the LVAD, in particular a much shorter diastolic resting phase compared with in vivo situations. Thus, the peak velocities and assumable temporal flow patterns are not identical. However, because of the moderate differences of in vivo and in vitro flow using the same PF-VAD,${ }^{21}$ it is assumed that the differences in the performance of the PF-VAD did not strongly influence the hemodynamic behavior of the whole model system with the additional retrograde flow support by the CF-VAD. Furthermore, a different compliance of the model (the synthetic elastic aorta and tubing) compared with in vivo situations may have an impact on pressure values. However, modifying the setup to accommodate these realities was outside the scope of this study. Additional measurements for test-retest reliability have not been conducted because a good agreement in reproducibility in in vitro 4D flow-sensitive MRI experiments using a VAD with a connected aorta model has been demonstrated recently. ${ }^{21}$

\section{CONCLUSIONS}

Our time-resolved 3D flow measurements have shown that despite the massive alterations in natural hemodynamics due to the retrograde flow via the right subclavian and brachiocephalic arteries, no drastic consequences on the flow characteristics in the aorta were observed. This is supported by recent experiences with partial-support devices for long-term-support in elderly patients with chronic heart failure. ${ }^{27,28}$

\section{Conflict of Interest Statement}

Drs Beyersdorf and Benk hold shares in ResuSciTec, Ltd. All other authors have nothing to disclose with regard to commercial support.

\section{References}

1. Park SJ, Tector A, Piccioni W, Raines E, Gelijns A, Moskowitz A, et al. Left ventricular assist devices as destination therapy: a new look at survival. J Thorac Cardiovasc Surg. 2005;129:9-17

2. Fang JC. Rise of the machines - left ventricular assist devices as permanent therapy for advanced heart failure. N Engl J Med. 2009;361:2282-5.

3. Long JW, Healy AH, Rasmusson BY, Cowley CG, Nelson KE, Kfoury AG, et al. Improving outcomes with long-term "destination" therapy using left ventricular assist devices. J Thorac Cardiovasc Surg. 2008;135:1353-61.

4. Caccamo M, Eckman P, John R. Current state of ventricular assist devices. Curr Heart Fail Rep. 2011;8:91-8.

5. Maltais S, Tchantchaleishvili V, Schaff HV, Daly RC, Suri RM, Dearani JA, et al. Management of severe ischemic cardiomyopathy: left ventricular assist device as destination therapy versus conventional bypass and mitral valve surgery. $J$ Thorac Cardiovasc Surg. 2014;147:1246-50.

6. John R, Mantz K, Eckman P, Rose A, May-Newman K. Aortic valve pathophysiology during left ventricular assist device support. Heart Lung Transplant. 2010; 29:1321-9.

7. Cowger J, Pagani FD, Haft JW, Romano MA, Aaronson KD, Kolias TJ. The development of aortic insufficiency in left ventricular assist device supported patients. Circ Heart Fail. 2010;3:668-74.

8. Toda K, Fujita T, Domae K, Shimahara Y, Kobayashi J, Nakatani T. Late aortic insufficiency related to poor prognosis during left ventricular assist device support. Ann Thorac Surg. 2011;92:929-34.

9. Hatano M, Kinugawa K, Shiga T, Kato N, Endo M, Hisagi M, et al. Less frequent opening of the aortic valve and a continuous flow pump are risk factors for postoperative onset of aortic insufficiency in patients with a left ventricular assist device. Circ J. 2011;75:1147-55.

10. Pak SW, Uriel N, Takayama H, Cappleman S, Song R, Colombo PC, et al. Prevalence of de novo aortic insufficiency during long-term support with left ventricular assist devices. J Heart Lung Transplant. 2010;29:1172-6.

11. Freed BH, Jeevanandam V, Jolly N. Aortic root and valve thrombosis after implantation of a left ventricular assist device. J Invasive Cardiol. 2011;23:63-5.

12. Crumpstone T, Martin TD, Yang JJ, Peng YG. Misplacement of LVAD inflow cannula leads to insufficient output and tissue hypoperfusion. J Artif Organs. 2010;13:225-7. 
13. Litwak KN, Koenig SC, Tsukui H, Kihara S, Wu Z, Pantalos GM. Effects of left ventricular assist device support and outflow graft location upon aortic blood flow. ASAIO J. 2004;50:432-7.

14. Karmonik C, Partovi S, Loebe M, Schmack B, Weymann A, Lumsden AB, et al. Computational fluid dynamics in patients with continuous-flow left ventricular assist device support show hemodynamic alterations in the ascending aorta. $J$ Thorac Cardiovasc Surg. 2014;147:1326-33.e1.

15. Laumen M, Kaufmann T, Timms D, Schlanstein P, Jansen S, Gregory S, et al. Flow analysis of ventricular assist device inflow and outflow cannula positioning using a naturally shaped ventricle and aortic branch. Artif Organs. 2010;34:798-806.

16. Kaufmann TA, Hormes M, Laumen M, Timms DL, Schmitz-Rode T, Moritz A, et al. Flow distribution during cardiopulmonary bypass in dependency on the outflow cannula positioning. Artif Organs. 2009;33:988-92.

17. Kar B, Delgado RM 3rd, Frazier OH, Gregoric ID, Harting MT, Wadia Y, et al. The effect of LVAD aortic outflow-graft placement on hemodynamics and flow: implantation technique and computer flow modeling. Tex Heart Inst J. 2005;32: 294-8.

18. May-Newman K, Hillen B, Dembitsky W. Effect of left ventricular assist device outflow conduit anastomosis location on flow patterns in the native aorta. ASAIO J. 2006;52:132-9.

19. Markl M, Benk C, Klausmann D, Stalder AF, Frydrychowicz A, Hennig J, et al. Three-dimensional magnetic resonance flow analysis in a ventricular assist device. J Thorac Cardiovasc Surg. 2007;134:1471-6.

20. Benk C, Lorenz R, Beyersdorf F, Bock J, Klemm R, Korvink JG, et al. Threedimensional flow characteristics in ventricular assist devices: impact of valve design and operating conditions. J Thorac Cardiovasc Surg. 2011;142:1019-26.

21. Lorenz R, Benk C, Bock J, Stalder AF, Korvink JG, Hennig J, et al. Closed circuit MR compatible pulsatile pump system using a ventricular assist device and pressure control unit. Magn Reson Med. 2012;67:258-68.
22. Benk C, Mauch A, Beyersdorf F, Klemm R, Russe M, Blanke P, et al. Effect of cannula position in the thoracic aorta with continuous left ventricular support: four-dimensional flow-sensitive magnetic resonance imaging in an in vitro model. Eur J Cardiothorac Surg. 2013;44:551-8.

23. Klotz S, Meyns B, Simon A, Wittwer T, Rahmanian P, Schlensak C, et al. Partial mechanical long-term support with the CircuLite Synergy pump as bridge-totransplant in congestive heart failure. Thorac Cardiovasc Surg 2010;58 Suppl 2:S173-8.

24. Meyns B, Klotz S, Simon A, Droogne W, Rega F, Griffith B, et al. Proof of concept: hemodynamic response to long-term partial ventricular support with the synergy pocket micro-pump. J Am Coll Cardiol. 2009;54:79-86.

25. Meyns BP, Simon A, Klotz S, Wittwer T, Schlensak C, Rega F, et al. Clinical benefits of partial circulatory support in New York Heart Association Class IIIB and Early Class IV patients. Eur J Cardiothorac Surg. 2011;39:693-8.

26. Fresiello L, Zieliński K, Jacobs S, Di Molfetta A, Pałko KJ, Bernini F, et al. Reproduction of continuous flow left ventricular assist device experimental data by means of a hybrid cardiovascular model with baroreflex control. Artif Organs. 2014;38:456-68.

27. Barbone A, Pini D, Rega F, Ornaghi D, Vitali E, Meyns B. Circulatory support in elderly chronic heart failure patients using the CircuLite Synergy system. Eur J Cardiothorac Surg. 2013;44:207-12.

28. Sabashnikov A, Popov AF, Bowles CT, Mohite PN, Weymann A, Hards R, et al. Outcomes after implantation of partial-support left ventricular assist devices in inotropic-dependent patients: Do we still need full-support assist devices? J Thorac Cardiovasc Surg. 2014;148:1115-21.

Key Words: aortic hemodynamics, 4D flow MRI, LVAD, outflow graft

\section{EDITORIAL COMMENTARY}

\section{Adding a new dimension to our understanding of continuous-flow physiology}

Nicholas G. Smedira, MD

See related article on pages 200-7.

In the United States from 2006 through 2013, approximately 10,000 continuous-flow left ventricular assist devices (CFVADs) were implanted. Data from the Interagency Registry for Mechanical Circulatory Support showed a 1-year

From the Department of Thoracic/Cardiovascular Surgery, The Cleveland Clinic Foundation, Cleveland, Ohio.

Disclosures: Author has nothing to disclose with regard to commercial support.

Received for publication April 15, 2015; accepted for publication April 15, 2015; available ahead of print May 16, 2015.

Address for reprints: Nicholas G. Smedira, MD, Department of Thoracic/Cardiovascular Surgery, The Cleveland Clinic Foundation, 9500 Euclid Ave, Desk F25,

Cleveland, OH 44195 (E-mail: smedirn@ ccf.org).

J Thorac Cardiovasc Surg 2015;150:207-8

$0022-5223 / \$ 36.00$

Copyright (C) 2015 by The American Association for Thoracic Surgery

http://dx.doi.org/10.1016/j.jtcvs.2015.04.031 mortality in patients bridged to transplant of only $12 \%$, and these results have led to the proposal that CF-VADs be considered as an alternative to transplantation. ${ }^{1,2}$ Despite this large clinical experience and these outstanding results, our understanding of the physiology of CF-VADs is extremely

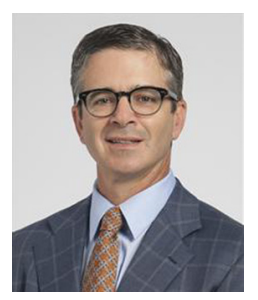
limited. As an example, it is very difficult to measure lowpulsatility blood pressure, and the safest long-term level of "mean" blood pressure is unknown.

In this issue of the Journal, Jung and colleagues ${ }^{3}$ from the University Hospital Bern report their elegant use of 4dimensional flow-sensitive magnetic resonance imaging to assess the impact of a CF-VAD on the flow characteristics in a mock circulation of the thoracic aorta and extracranial cerebral vessels. They and their reviewers note some of the limitations, including the use of a pulsatile device as the heart, the fixed uniform resistance across multiple arteries, 